NSF-ITP-99-020

hep-th/9903227

\title{
Holographic description of D3-branes in flat space
}

\author{
Akikazu Hashimoto \\ Institute for Theoretical Physics \\ University of California, Santa Barbara, CA 93106 \\ aki@itp.ucsb.edu
}

\begin{abstract}
We describe a scheme for constructing the holographic dual of the full D3-brane geometry with charge $K$ by embedding it into a large anti-de Sitter space of size $N$. Such a geometry is realized in a multi-center anti-de Sitter geometry which admits a simple field theory interpretation as $S U(N+K)$ gauge theory broken to $S U(N) \times S U(K)$. We find that the characteristic size of the D3-brane geometry is of order $(K / N)^{1 / 4} U^{0}$ where $U^{0}$ is the scale of the Higgs. By choosing $N$ to be much larger than $K$, the scale of the D3-brane metric can be well separated from the Higgs scale in the radial coordinate. We generalize the holographic energy-distance relation and estimate the characteristic energy scale associated with these radial scales, and find that the $E / U$ relation becomes effectively $U$ independent in the range $(K / N)^{1 / 2} U^{0}<U<U^{0}$. This implies that all detailed structure of the D3-brane geometry is encoded in the fine structure of the boundary gauge theory at around the Higgs scale.
\end{abstract}

March 1999 
The conjecture of Maldacena [1] relating type IIB string theory on $A d S_{5} \times S_{5}$ to $\mathcal{N}=4$ SYM on $3+1$ dimensions was originally formulated by taking the "near horizon" or the "decoupling" limit of D3-branes sitting in flat space. Even before the formulation of the Maldacena conjecture, the close connection between the gauge theory living on the world volume of a D-brane and the supergravity background was explored in the context of computing absorption cross-sections [2, 3, 4]. Once the details of the correspondence between the bulk and the boundary theories were better understood [5, 6], it became sensible to discard the asymptotically flat region of the D3-brane background and work entirely with the near horizon anti-de Sitter geometry. The reason is that the Maldacena conjecture can be stated concretely in a form of an identity of suitably defined generating functions between string theory on anti-de Sitter space and the gauge theory on its boundary. One speaks of the boundary theory being "holographically dual" to the bulk theory $[7,8]$, and in the case of $A d S_{5} \times S_{5}$ arising from the near horizon limit of the D3-brane, both the bulk ${ }^{1}$ and the boundary theory are well defined.

Just because we have come to understand the holographic dual of the near horizon $A d S_{5} \times S_{5}$ geometry does not mean that the holographic dual of the full D3-brane geometry does not exist. Indeed, we expect all theory of gravity to have a holographic dual $[7,8]$. It is therefore quite natural to ask what the boundary dual to string theory in the full D3-brane background might be. In this note we provide an answer to this question, although in an implicit form.

It is easy to appreciate the scope of this problem: the holographic dual on the boundary is likely to be a very complicated theory. The D3-brane background and the $A d S_{5} \times S_{5}$ background behave more or less the same way in the small radius region, but differ drastically at large radius. In holography, small and large radius regions of the bulk theory are associated with the infra-red and ultra-violet of the boundary theory, respectively $[1,9]$. This means that the UV structure of the boundary theory must be drastically different than that of the SYM theory. In [10], it was suggested that this theory might be realized as a condensate of the irrelevant operator $F^{4}$. However, without the detailed knowledge of the UV structure of the theory, it is not clear if a sensible meaning can be attached to condensation of an irrelevant operator (see e.g. [6]). Since the geometry of the D3-brane metric is asymptotically flat, it is quite likely that the boundary theory, whatever it may be, is as complicated as the holographic dual of the full type IIB string theory on Minkowski space $[11,12,13]$.

In this note, we resolve the ambiguity of the UV structure of the boundary theory by embedding it in some other theory whose UV structure is better understood. The boundary

\footnotetext{
${ }^{1}$ This is true up to the subtle issue of defining string theories in a presence of RR background. Currently, only the supergravity approximation which can be trusted at low energies is properly formulated.
} 
theory of interest to us can be extracted by flowing along the renormalization group down to the appropriate scale. Although such a procedure will always lead to a UV complete description of an effective field theory, it is generally difficult to determine what UV fixed point can give rise to a renormalization group flow that reaches the effective theory of interest. In a holographic theory, however, it is very straight forward to embed an arbitrary IR structure of interest into a well defined UV structure. The idea is to simply embed the bulk geometry of the IR theory somewhere in the near core region which smoothly interpolates to the bulk geometry of the UV fixed point. A very convenient bulk geometry to use as a UV fixed point is the anti-de Sitter space, since we understand the UV structure from the boundary point of view as well ${ }^{2}$. In order to formulate the holographic dual of the D3-brane geometry, all that we have to do is to consider a bulk geometry which at small radius looks more or less like a D3-brane geometry, but behaves at very large radius like an anti-de Sitter space.

It turns out that a supergravity solution which interpolates between a full D3-brane metric (with the asymptotically flat region) and an anti-de Sitter space is very easy to construct. One needs to look no further than the multi-centered anti-de Sitter solution. Let us demonstrate this explicitly. Consider a type IIB supergravity solution for parallel D3-branes with charges $N \gg K \gg 1$.

$$
d s^{2}=\left(1+\frac{4 \pi g N \alpha^{\prime 2}}{\left(\vec{x}_{\perp}+\vec{x}_{\perp}^{0}\right)^{4}}+\frac{4 \pi g K \alpha^{\prime 2}}{\vec{x}_{\perp}^{4}}\right)^{-1 / 2} d \vec{x}_{\|}^{2}+\left(1+\frac{4 \pi g N \alpha^{\prime 2}}{\left(\vec{x}_{\perp}+\vec{x}_{\perp}^{0}\right)^{4}}+\frac{4 \pi g K \alpha^{\prime 2}}{\vec{x}_{\perp}^{4}}\right)^{1 / 2} d \vec{x}_{\perp}^{2} .
$$

Taking the decoupling limit keeping $\vec{U}_{\perp}=\vec{x}_{\perp} / \alpha^{\prime}$ fixed gives

$$
d s^{2}=\alpha^{\prime}\left(\frac{4 \pi g N}{\left(\vec{U}_{\perp}+\vec{U}_{\perp}^{0}\right)^{4}}+\frac{4 \pi g K}{\vec{U}_{\perp}^{4}}\right)^{-1 / 2} d \vec{x}_{\|}^{2}+\alpha^{\prime}\left(\frac{4 \pi g N}{\left(\vec{U}_{\perp}+\vec{U}_{\perp}^{0}\right)^{4}}+\frac{4 \pi g K}{\vec{U}_{\perp}^{4}}\right)^{1 / 2} d \vec{U}_{\perp}^{2} .
$$

If we restrict to range of parameters $|\vec{U}| \approx(k / N)^{1 / 4}\left|\vec{U}_{\perp}^{0}\right| \ll\left|\vec{U}_{\perp}^{0}\right|$, the metric simplifies to

$$
d s^{2}=\alpha^{\prime} \frac{\left|\vec{U}_{\perp}^{0}\right|^{2}}{\sqrt{4 \pi g N}}\left(1+\frac{(K / N)\left|\vec{U}_{\perp}^{0}\right|^{4}}{|\vec{U}|^{4}}\right)^{-1 / 2} d \vec{U}_{\|}^{2}+\alpha^{\prime} \frac{\sqrt{4 \pi g N}}{\left|\vec{U}_{\perp}^{0}\right|^{2}}\left(1+\frac{(K / N)\left|\vec{U}_{\perp}^{0}\right|^{4}}{|\vec{U}|^{4}}\right)^{1 / 2} d \vec{U}_{\perp}^{2}
$$

Finally, a simple rescaling

$$
\vec{y}_{\|}=\frac{\sqrt{\alpha^{\prime}}\left|\vec{U}_{\perp}^{0}\right|}{(4 \pi g N)^{1 / 4}} \vec{x}_{\|}, \quad \vec{r}_{\perp}=\frac{\left(4 \pi g N \alpha^{2}\right)^{1 / 4}}{\left|\vec{U}_{\perp}^{0}\right|} \vec{U}_{\perp}
$$

leads to the standard from of the D3-brane metric

$$
d s^{2}=\left(1+\frac{4 \pi g K \alpha^{2}}{r^{4}}\right)^{-1 / 2} d \vec{y}_{\|}^{2}+\left(1+\frac{4 \pi g K \alpha^{2}}{r^{4}}\right)^{1 / 2} d \vec{r}_{\perp}^{2} .
$$

\footnotetext{
${ }^{2}$ Geometric embeddings of an IR dynamics in some UV theory utilizing the holographic principle was discussed in $[14,15,16,17]$.
} 
Essentially, the $4 \pi g N /\left(\vec{U}_{\perp}+\vec{U}_{\perp}^{0}\right)^{4}$ term in the harmonic function at the vicinity of $U=0$ acts as the "1" to simulate the asymptotically flat region. An important point that we learn from this exercise is that the characteristic length scale $(K / N)^{1 / 4}\left|\vec{U}_{\perp}^{0}\right|$ associated with the D3-brane metric is clearly well separated from the Higgs scale $\left|\vec{U}_{0}^{\perp}\right|$ or the scale of the cosmological curvature $(4 \pi g N)^{1 / 4} / \alpha^{1 / 2}$. Therefore, there is plenty of room to fit an entire D3-brane metric with an asymptotically flat region, especially if we take the large $N$ limit while focusing on scales of order $(K / N)^{1 / 4}\left|\vec{U}_{\perp}^{0}\right|$. This separation of scales was possible because we chose to scale $K$ and $N$ differently.

In a certain sense, the point being made here is rather trivial in light of the fact that one takes a large $N$ limit of the anti-de Sitter space with RR charge $N$ to recover the flat space $[12,13]$. If we combine such a flat space limit with a source for $K$ units of RR flux, we were bound to recover the full D3-brane metric.

The interpretation of multi-centered anti-de Sitter solution as the SYM with Higgs VEV of order $\vec{U}_{\perp}^{0}$ have been discussed by many authors $[1,18,19]$. One surprising aspect of SYM with large 't Hooft coupling is the fact that energies associated with this VEV is not $\left|\vec{U}_{\perp}^{0}\right|$ but $\left|\vec{U}_{\perp}^{0}\right| / \sqrt{g N}[20]$. In other words, gauge theory observable such as the two point function $\langle\mathcal{O}(x) \mathcal{O}(0)\rangle$ will only feel the effect of Higgsing when $1 / x$ becomes of order $\left|\vec{U}_{\perp}^{0}\right| / \sqrt{g N}$. Having identified $(K / N)^{1 / 4}\left|\vec{U}_{\perp}^{0}\right|$ as an important scale in describing the D3-brane geometry, we would like to know to what energy this scale corresponds from the point of view of the boundary theory. We will address this issue in the remainder of this note.

In order to associate an energy scale to $(K / N)^{1 / 4}\left|\vec{U}_{\perp}^{0}\right|$, we need to generalize the notion of "energy-distance" relation

$$
E=\frac{U^{(5-p) / 2}}{g_{Y M} \sqrt{N}},
$$

derived in $[9,20]$, to multi-center backgrounds. This formula was derived in [20] by computing the "skin depth" of the boundary fluctuation into the bulk, and by utilizing the holographic information bound in [9]. It turns out, however, that this same formula can be derived in many different ways. ${ }^{3}$ We will use the method most convenient for us, but let us list a few example of an alternative method for deriving (6).

One such approach is to recall the calculation of Wilson loop expectation value as minimal surfaces $[21,22]$. When a string is suspended from infinity with its endpoint separated by $L=1 / E$, it penetrates the radial direction down to some radius $U$. The relation between $U$ and $L=1 / E$ is equivalent to $(6)^{4}$. Perhaps the methods developed in [23] can be adopted for this purpose.

\footnotetext{
${ }^{3}$ We thank Sunny Itzhaki for many discussions on this point.

${ }^{4}$ See equation $(5.2)$ of $[22]$.
} 
An alternative approach is to consider a light-cone originating from a fixed point in the boundary [24]. In a given time $t$, the signal on the boundary will spread in size by the rate determined by the speed of light. The energy associated with this size is therefore $E=1 / t$. In the same time interval, the light signal will travel in the radial direction by some amount $U(t)$. The relation between $U$ and $E$ determined this way also leads to (6).

For our application, this last approach is very convenient since all that is required is to solve the null geodesic equation

$$
d t=\left(\frac{4 \pi g N}{\left(U+U^{0}\right)^{4}}+\frac{4 \pi g K}{U^{4}}\right)^{1 / 2} d U
$$

where for convenience, we have oriented $\vec{U}$ to be parallel to $\vec{U}^{0}$ and dropped the vector sign. Choosing different orientations will not drastically change the story as long as the signal do not fall into the region near the $N$ branes. Note that if we set $U^{0}=0$ and $K=0$, this equation can be integrated trivially to give

$$
t=\frac{\sqrt{4 \pi g N}}{U}=\frac{1}{E}
$$

which is equivalent to (6). If $U^{0}$ and $K$ are both non-vanishing, we will have to work a little harder. To get a rough estimate of $t(U)$, it is convenient to divide the parameter space for $U$ into regions: (a) $U^{0}<U$, (b) $(K / N)^{1 / 4} U^{0}<U<U^{0}$, and (c) $U<(K / N)^{1 / 4} U^{0}$. In region (a), we might as well set $U^{0}=0$ and we get the essentially same result as (8)

$$
t(U)=\frac{\sqrt{4 \pi g(N+K)}}{U} \approx \frac{\sqrt{4 \pi g N}}{U} .
$$

In region (b), (7) can be approximated by

$$
d t=\frac{\sqrt{4 \pi g N}}{\left(U^{0}\right)^{2}} d U
$$

so we find

$$
t(U)=\frac{\sqrt{4 \pi g N}}{\left(U^{0}\right)^{2}}\left(U^{0}-U\right)+t\left(U^{0}\right)=\frac{\sqrt{4 \pi g N}}{U^{0}}\left(2-\frac{U}{U^{0}}\right) .
$$

Finally, in region (c), (7) can be approximated by

$$
d t=\frac{\sqrt{4 \pi g K}}{U^{2}} d U
$$

so we find

$$
t(U)=\sqrt{4 \pi g K}\left(\frac{1}{U}-\frac{1}{(K / N)^{1 / 4} U^{0}}\right)+t\left((K / N)^{1 / 4} U^{0}\right)=\frac{\sqrt{4 \pi g K}}{U}+\frac{\sqrt{4 \pi g N}}{U^{0}}\left(2-2(K / N)^{1 / 4}\right) .
$$




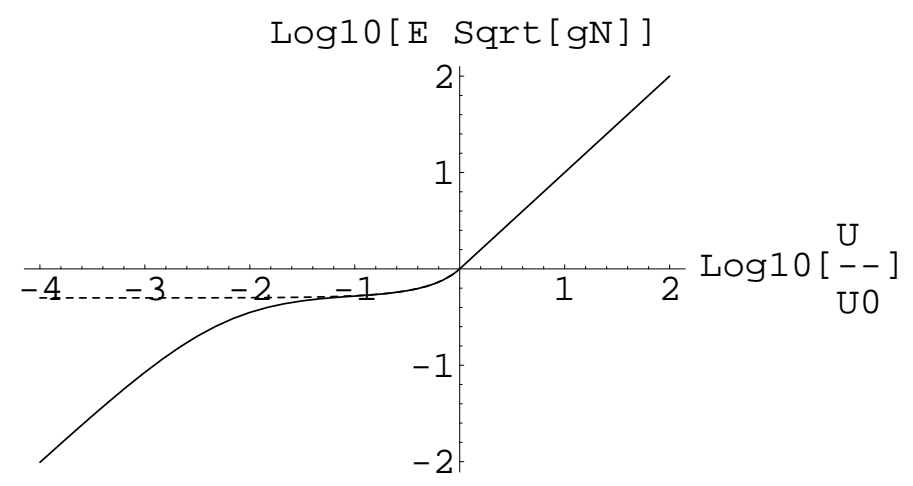

Figure 1: $\log -\log$ plot of the $E / U$ function for the multi-centered $A d S_{5} \times S_{5}$. The solid line is for $K / N=10^{-4}$. The dashed line is for $K / N=0$.

Inverting (9), (11), and (13), we seem to be finding that in region (a),

$$
E(U) \approx \frac{U}{\sqrt{4 \pi g N}}
$$

In region (b) by the time $U$ reaches $(K / N)^{1 / 4} U^{0}, E(U)$ is given by

$$
E(U) \approx \frac{1}{2} \frac{U^{0}}{\sqrt{4 \pi g N}}\left(1+\frac{1}{2}\left(\frac{K}{N}\right)^{1 / 4}\right),
$$

which is of the same order of magnitude as $E\left(U^{0}\right)$. In fact, $E(U)$ will continue to be in the same order of magnitude even in region (c) until $U$ become smaller than $\sqrt{K / N} U^{0}$ at which point the first term in (13) starts to dominate, leading to

$$
E(U)=\frac{U}{\sqrt{4 \pi g K}} .
$$

Since we do not rely on this kind of analysis beyond estimating orders of magnitudes, the picture that seems to be emerging is the following: the "energy-distance" relation for $U>U^{0}$ behaves like (14), while for $U<\sqrt{K / N} U^{0}$ it behaves like (16). For the values of $U$ in between these regions, $E(U)$ will remain more or less constant at the energy of order Higgs scale $E=U / \sqrt{g N}$. Note that the scale of interest to us, namely $U \approx(K / N)^{1 / 4} U^{0}$ is precisely in this flat region. See figure 1 for an illustration.

The multi-center solution breaks the $S_{5}$ spherical symmetry, but this symmetry can be restored by smearing the supergravity solution (2) along the $S_{5}$. This leads to a geometry of the form

$$
d s^{2}=\alpha^{\prime} f(U)^{-1 / 2} d \vec{x}_{\|}^{2}+\alpha^{\prime} f(U)^{1 / 2} d \vec{U}^{2} .
$$

where

$$
f(U)= \begin{cases}\frac{4 \pi g(N+K)}{U^{4}} & \left(U^{0}<U\right) \\ \frac{4 \pi g N}{\left(U^{0}\right)^{4}}\left(1+\frac{K\left(U^{0}\right)^{4}}{N U^{4}}\right) & \left(U<U^{0}\right)\end{cases}
$$


which is that of an $A d S_{5} \times S_{5}$ for $U>U^{0}$ and a full D3-brane geometry of size $(K / N)^{1 / 4} U^{0}$ for $U<U^{0}$. If we set $K=0$, this is precisely the flat space region described in [25]. Turning on $K$ then has a simple interpretation as placing a small D3-brane in this flat space-time patch. One can apply the method of $[5,6]$ for computing the correlation function of chiral primary operators by solving the wave equation for modes propagating in this background. At linear order in fluctuations, the minimal scalar equation will be of the standard Bessel form for $U^{0}<U$, whereas for $U<U^{0}$, it will be of the Mathieu form [26]. The solution to these equation are to be matched at $U=U^{0}$. The energy distance relation can be derived using the null geodesic equation and, for non-zero $K$, leads to the same general structure as what we found for the unsmeared solution ${ }^{5}$.

This conclusion seems rather robust and independent of the details of the derivation. If we insist that (a) $E(U)$ be given by (14) down to $U=U^{0},(\mathrm{~b})$ that $E(U)$ to be given by (16) deep in the IR, and (c) that $E(U)$ be a monotonic function, then $E(U)$ must remain more or less flat in the region $\sqrt{K / N} U^{0}<U<U^{0}$.

We seem to have no choice but to conclude that although $U^{0}$ and $(K / N)^{1 / 4} U^{0}$ appear to be well separated in the radial scale, from the point of view of the energy via the "energydistance" relation, the two scales are not separated at all. In fact, they are all at the Higgs scale. It seems quite remarkable that the detailed structure of full D3-brane metric is encoded in the "fine structure" of the Higgsed SYM at around the Higgs scale. With a sufficiently powerful spectrum analyzer and with a lot of patience, all of the details of say the absorption and Hawking emission by the D3-brane including all Mathieu [26] and quantum corrections is in principle reconstructible from the gauge theory. From a practical point of view, however, the fact that so much information is crammed in such a narrow scale in energy suggests that it would be extremely difficult to isolate and extract information of interest from the full theory.

\section{Acknowledgements}

I would like to thank Steve Gubser, Sunny Itzhaki, Finn Larsen, and Joe Polchinski for illuminating discussions. This work was supported in part by the National Science Foundation under Grant No. PHY94-07194.

\footnotetext{
${ }^{5}$ It is interesting to note that for $K=0$, the energy converges to a finite quantity of order $U^{0} / \sqrt{g N}$ as $U \rightarrow 0$. This suggests that the theory is empty at energies below this scale. In fact, the field theory dual to this simple background seems to exhibit mass-gap and confinement.
} 


\section{References}

[1] J. Maldacena, "The Large N limit of superconformal field theories and supergravity," Adv. Theor. Math. Phys. 2 (1998) 231, hep-th/9711200.

[2] I. R. Klebanov, "World volume approach to absorption by nondilatonic branes," Nucl. Phys. B496 (1997) 231, hep-th/9702076.

[3] S. S. Gubser, I. R. Klebanov, and A. A. Tseytlin, "String theory and classical absorption by three-branes," Nucl. Phys. B499 (1997) 217, hep-th/9703040.

[4] S. S. Gubser and I. R. Klebanov, "Absorption by branes and Schwinger terms in the world volume theory," Phys. Lett. B413 (1997) 41-48, hep-th/9708005.

[5] S. S. Gubser, I. R. Klebanov, and A. M. Polyakov, "Gauge theory correlators from noncritical string theory," Phys. Lett. B428 (1998) 105, hep-th/9802109.

[6] E. Witten, "Anti-de Sitter space and holography," Adv. Theor. Math. Phys. 2 (1998) 253, hep-th/9802150.

[7] G. 't Hooft, "Dimensional reduction in quantum gravity," gr-qc/9310026.

[8] L. Susskind, "The World as a hologram," J. Math. Phys. 36 (1995) 6377-6396, hep-th/9409089.

[9] L. Susskind and E. Witten, "The Holographic bound in anti-de Sitter space," hep-th/9805114.

[10] S. S. Gubser, A. Hashimoto, I. R. Klebanov, and M. Krasnitz, "Scalar absorption and the breaking of the world volume conformal invariance," Nucl. Phys. B526 (1998) 393, hep-th/9803023.

[11] E. Witten, talk presented at Strings 98 Conference, http://www.itp.ucsb.edu/online/strings98/witten/.

[12] J. Polchinski, "S matrices from AdS space-time," hep-th/9901076.

[13] L. Susskind, "Holography in the flat space limit," hep-th/9901079.

[14] N. Itzhaki, J. M. Maldacena, J. Sonnenschein, and S. Yankielowicz, "Supergravity and the large N limit of theories with sixteen supercharges," Phys. Rev. D58 (1998) 046004, hep-th/9802042. 
[15] L. Girardello, M. Petrini, M. Porrati, and A. Zaffaroni, "Novel local CFT and exact results on perturbations of $\mathrm{N}=4$ superYang Mills from AdS dynamics," JHEP 12 (1998) 022, hep-th/9810126.

[16] J. Distler and F. Zamora, "Nonsupersymmetric conformal field theories from stable anti-de Sitter spaces," hep-th/9810206.

[17] M. Porrati and A. Starinets, "RG fixed points in supergravity duals of 4-D field theory and asymptotically AdS spaces," hep-th/9903085.

[18] M. R. Douglas and W. Taylor, "Branes in the bulk of Anti-de Sitter space," hep-th/9807225.

[19] Y.-Y. Wu, "A Note on AdS/SYM correspondence on the Coulomb branch," hep-th/9809055.

[20] A. W. Peet and J. Polchinski, "UV/IR relations in AdS dynamics," Phys. Rev. D59 (1999) 065006, hep-th/9809022.

[21] J. Maldacena, "Wilson loops in large N field theories," Phys. Rev. Lett. 80 (1998) 4859, hep-th/9803002.

[22] A. Brandhuber, N. Itzhaki, J. Sonnenschein, and S. Yankielowicz, "Wilson loops, confinement, and phase transitions in large N gauge theories from supergravity," JHEP 06 (1998) 001, hep-th/9803263.

[23] J. A. Minahan and N. P. Warner, "Quark potentials in the Higgs phase of large N supersymmetric Yang-Mills theories," JHEP 06 (1998) 005, hep-th/9805104.

[24] G. T. Horowitz and N. Itzhaki, "Black holes, shock waves, and causality in the AdS/CFT correspondence," hep-th/9901012.

[25] P. Kraus, F. Larsen, and S. P. Trivedi, "The Coulomb branch of gauge theory from rotating branes," hep-th/9811120.

[26] S. S. Gubser and A. Hashimoto, "Exact absorption probabilities for the D3-brane," hep-th/9805140. 